\title{
2036 Acute alcohol-induced myocardial inflammation as visualized by cardiac magnetic resonance
}

\author{
Anja Zagrosek*, Steffen Bohl, Ralf Wassmuth, André Rudolph, \\ Daniel Messroghli, Rainer Dietz and Jeanette Schulz-Menger
}

Address: Franz-Volhard-Clinic, Charité-Campus Buch, Helios-Klinikum, Humboldt-University, Berlin, Germany

* Corresponding author

from I th $^{\text {th }}$ Annual SCMR Scientific Sessions

Los Angeles, CA, USA. I-3 February 2008

Published: 22 October 2008

Journal of Cardiovascular Magnetic Resonance 2008, I0(Suppl I):A305 doi:I0.I I86/I532-429X-I0-SI-A305

This abstract is available from: http://jcmr-online.com/content//0/SI/A305

(c) 2008 Zagrosek et al; licensee BioMed Central Ltd.

\section{Introduction}

Intoxication with large amounts of ethanol is known to induce a systemic, inflammatory reaction including excretion of several cytokines, most often leading to the socalled "hangover". We hypothesised that the systemic inflammatory imbalance leads to a transient sterile myocardial inflammation which can be visualized by cardiac magnetic resonance (CMR) employing the techniques established for the diagnosis of inflammatory myocardial injury.

\section{Methods}

In 10 healthy volunteers (age mean \pm SD $31.6 \pm 2.8$ yrs., BMI $22.6 \pm 2.0,3$ female) a hangover was induced experimentally by consumption of large amounts of vodka. After 1 week of abstinence from alcohol, participants were examined in a $1.5 \mathrm{~T}$ CMR-System before, $12 \mathrm{~h}$ and 1 week after alcohol intake. The CMR protocol included cineimaging for cardiac function and volumes, T2-weighted techniques for detection of cardiac edema (T2-ratio: ratio of signal intensitiy of myocardium/sceletal muscle), "early" global relative enhancement (gRE, axial spin-echo sequence comparing myocardial and sceletal uptake of contrast agent) and late hyperenhancement for detection of myocardial scarring. Blood alcohol level (BAL) was assessed $45 \mathrm{~min}$. after termination of alcohol intake. The study was approved by the local ethics committee.

\section{Results}

All participants suffered from a clinical "hangover" $12 \mathrm{~h}$ after alcohol intake (headache, nausea, fatigue, tremulousness, tachycardia). The mean BAL at "top-of-drinking" was $1.1 \pm 0.3 \%$ o $(1.4 \pm 0.4 \mathrm{~g} / \mathrm{l})$. In CMR, a significant increase in signal intensity on T2-weighted images was observed on the day after drinking (T2-ratio $1.7 \pm 0.2$ vs. $1.9 \pm 0.2, \mathrm{p}=0.04)$. In 8 participants $(80 \%)$ gRE after drinking was significantly enhanced as well $(3.9 \pm 1.3$ vs. $6.8 \pm 3.1, \mathrm{p}=0.03)$. The CMR-parameters returned to baseline values within 1 week after alcohol intake in 9 participants $(90 \%)$. Left ventricular function and volumes remained unchanged during all three CMR exams. On late hyperenhancement images, no areas of focal necrosis were detectable.

\section{Conclusion}

This study supports the hypothesis that even moderate alcohol-intoxication leads to a transient myocarditis-like inflammation of the myocardium which is detectable by CMR. Larger studies are required to verify the present findings. 